\title{
Práctica de la respiración profunda para el estrés académico en estudiantes de la Universidad Nacional de Educación
}

\section{Deep breathing practice for academic stress in students at the National University of Education}

\author{
Ivonne Lorena Carpio Toro ${ }^{1}$ \\ ${ }^{1}$ Universidad Catolica de Cuenca, Ecuador \\ *ivoncarpiot@hotmail.com
}

DOI: https://doi.org/10.26871/killkana_salud.v4i3.602

\begin{abstract}
Resumen
Contexto: Los estudiantes universitarios enfrentan exigencias académicas y psicosociales en su experiencia escolar, lo que genera un estado acumulativo/progresivo de estrés durante los años que dura este período, y esta situación desencadena consecuencias negativas para su bienestar, tanto físicas como psíquicas. Objetivo: El objetivo de este estudio fue examinar la efectividad de la respiración yóguica (técnicas que procuran rangos de respiración de entre 4 y 8 repeticiones por minuto), mediante un cuasiexperimento con medidas pre-post y grupo de control, además de un análisis del discurso sobre la producción textual realizada, después de experimentar las respiraciones referidas por los estudiantes de la Universidad Nacional de Educación en Ecuador. Metodología: Se utilizó el Cuestionario de Estrés Académico en la Universidad (CEAU) en una muestra conformada por 73 estudiantes de ambos sexos, con edad media de $19 \pm 2$ años, seleccionados de la Carrera de Educación General Básica, de primer ciclo. Resultados: Se evidenció, a través del cuestionario y del análisis del discurso aplicado, que la intervención produjo una reducción del nivel de estrés. Se pudo observar que las medias de las escalas del test CEAU en el grupo de control pre y post test se mantuvieron o elevaron, a diferencia del grupo de intervención en los que disminuyeron los niveles de estrés. Conclusiones: Una práctica regular de las técnicas de pranayama, en el contexto áulico, son de mucha utilidad en la reducción del estrés académico.
\end{abstract}

Palabras clave: Respiración profunda, pranayama, educación, universidad, Ecuador.

\begin{abstract}
Context: University students face academic and psychosocial demands in their school experience, which generates a cumulative / progressive state of stress during the years that this period lasts, unleashing negative consequences for their well-being, both physical and mental. Objective: The objective of this study was to examine the effectiveness of yogic breathing (techniques that seek breathing ranges of between 4 and 8 repetitions per minute), through a quasi-experiment with pre-post and control group measurements, in addition to an analysis of the discourse on the textual production carried out, after experiencing the aforementioned breaths, by the students of the National University of Education in Ecuador. Methodology: The University Academic Stress Questionnaire (CEAU) was used in a sample made up of 73 students of both sexes, with a mean age of $19 \pm 2$ years, selected from the Basic General Education Career, first cycle. Results: It was evidenced, through the questionnaire and the analysis of the applied discourse, that the intervention produced a reduction in the level of stress. It was observed that the means of the CEAU test scales in the pre and post-test control group were maintained or raised, unlike the intervention group in which stress levels decreased. Conclusions: A regular practice of pranayama techniques, in the classroom context, are very useful in reducing academic stress.
\end{abstract}

Keywords: Deep breathing, pranayama, education, university, Ecuador.

\section{Introducción}

El estrés es un fenómeno biopsicosocial que produce alteraciones en la salud física, mental y por tanto un potencial generador de patologías; ${ }^{1}$ por lo que es muy estudiando en la contemporaneidad, debido a las diferentes demandas como la exigencia, la competitividad laboral o académica y la excesiva cantidad de información a la que estamos expuestos.

En la educación, el estrés académico, es evidente con un $43.9 \%$ de prevalencia, siendo la tercera causa de consulta después de la depresión y la ansiedad. ${ }^{2}$ Además se observa como la principal dificultad en relación al rendimiento académico y las relaciones interpersonales ${ }^{3}$ en diferentes investigaciones se han encontrado desde niveles leves a 
moderados de estrés y cansancio emocional ${ }^{4}$ hasta niveles altos, ${ }^{5}$ y que también se han relacionado con la depresión y la ansiedad. , $^{6}$

El estés académico está presente en los estudiantes ecuatorianos $;^{8,9}$ en Guayaquil el nivel de estrés observado en los estudiantes es medio $(73,53 \%)$ lo que indica presencia de ansiedad, angustia, inquietud, cansancio permanente, somnolencia, dolores de cabeza, además los elementos más importantes que la originan son, las demandas del entorno, en la que el docente, la metodología, la carga, los exámenes y el tiempo son elementos de importante incidencia en el desarrollo de este fenómeno. ${ }^{10}$

En Riobamba se detectó igualmente que el $65 \%$ de la muestra tenía un nivel de estrés leve y el $13 \%$ moderado. En más del $50 \%$ de los casos hubo reacciones psicológicas como pérdida de la concentración, preocupación excesiva, depresión, inquietud, nerviosismo, ansiedad; reacciones físicas como cansancio, fatiga, poco o mucho deseo de alimentarse y reacciones de comportamiento como aislamiento y alteraciones en el sueño ${ }^{11}$

Siguiendo este orden de ideas, en las unidades educativas de Cuenca, en alrededor de un $80 \% .{ }^{12}$ Dentro de las consecuencias más relevantes están las respuestas psicológicas como ira, ansiedad, desesperanza y baja autoestima; además posee un componente principal cognitivo que afecta las ideas y pensamientos ya que el estímulo externo representa una amenaza para el individuo (la cual dependerá, en su gradación, de la personalidad y el nivel de soporte social de dicho individuo) y su capacidad para afrontar dicha realidad. $1,7,13$

Por lo tanto, es imperativo generar estrategias tanto para estudiantes como para los docentes para concientizar, discutir y evidenciar dicha problemática y, además, herramientas que permitan trabajar esa realidad en las aulas, debido a que son pocas alternativas propuestas.

Por lo dicho, se propone una investigación cuasiexperimental con la hipótesis basada en que la respiración profunda reduce el nivel de estrés en los estudiantes de primer ciclo de la Carrera de Educación General Básica de la Universidad Nacional de Educación del Ecuador (UNAE).

\section{Marco conceptual}

La educación, dentro de los retos de la contemporaneidad, implica el uso de herramientas que le permitan al educando un desarrollo crítico de su entendimiento sobre las realidades y de su participación en las mismas y, estas destrezas, se alcanzan a través de un trabajo holístico que integra diferentes maneras de concebir el aprendizaje y las relaciones que en él se desarrollan.

Es casi un lugar común, en el contexto universitario, hablar de la alta carga de tareas, obligaciones y responsabilidades que deben afrontar los estudiantes: exigencias sobre el rendimiento, intervenciones en público, clima social negativo, exámenes, dificultades de participación; así como a la presión que ejercen los padres, compañeros, profesores y ellos mismos, se desencadenan factores generadores de estrés que pueden afectar el rendimiento y la salud $\mathrm{d}^{6,12,13}$

De esta manera, el estrés académico se vincula a la valoración que realizan los estudiantes de las diferentes demandas o estresores de su ambiente, que desequilibran su medio interno, así como de las estrategias de afrontamiento para restablecer la homeostasis(Lemos et al., 2018; Quito et al., 2017). Se evidencia a través de una fase inicial de alarma, con signos como el aumento de presión, ritmo cardiaco, respiración, etc.; una segunda fase de adaptación o resistencia, cuando los estresores se mantienen en el tiempo y, finalmente, una fase de agotamiento en la cual se presentan reacciones como cansancio, dolor muscular, dificultad para concentrarse, irritabilidad, entre otras. ${ }^{1}$

En tal sentido, la educación debe atender los procesos físicos-psicológicos-emocionales que marcan el discurso que significa la educación en nuestra sociedad, sobre todo en lo referente al estrés académico en cuanto realidad que se vive en las instituciones educativas y, en esta búsqueda, nos posicionamos a partir del diálogo de saberes y la interculturalidad que le permiten a una episteme establecer contactos con otras maneras de construir el conocimiento para, en el discurso educativo, hablar de respiración yóguica (en adelante: pranayama) en cuanto herramienta que cataliza procesos fisiológicos y mentales hacia estados de introspección en cuanto formas de desarrollo intelectual (por la capacidad de concentración que dichos procesos generan) $\mathrm{y}$, sobre todo, de desarrollo emocional, que le permiten al sujeto mantener una relación armónica consigo mismo y con su ambiente educacional-social.

La ciencia occidental ha estudiado los efectos del yoga y la respiración en sus diferentes modalidades, y han demostrado que la intervención sobre la mente y el cuerpo, a través de prácticas respiración profunda, son efectivas al tratar desordenes físicos y mentales ocasionados por el estrés, ${ }^{14}$

Desde el kundalini yoga se busca potenciar la salud física, mental y emocional del ser humano en cuanto la energía del sistema glandular se combina con la energía del sistema nervioso y propone el uso de posturas físicas (asanas), el uso del sonido (mantra) y de formas de respiración (pranayamas). ${ }^{15}$ Para generar bienestar a través del entendimiento y manejo de la mente, la respiración (ciencia del uso del prana) es una constante que tiene una tradición milenaria, y se considera un factor crucial para el ser humano ${ }^{16}$

En la revisión sistemática de Zaccaro y colaboradores (2018), reconocen que los principales efectos de las técnicas de respiración lenta abarcan las actividades del sistema nervioso central y autónomo, así como el estado psicológico, y que si se establece ritmos respiratorios de 10 inhalaciones/exhalaciones por minuto, se pueden evidenciar impactos directos sobre la salud física y emocional de los sujetos que la realizan.

Yogi Bhajan menciona que con la técnica del pranayama la mente se detiene automáticamente cuando comienza 
la respiración mecánica rítmica, se tratará de 3 técnicas respiratorias que procuran la respiración lenta y profunda -que alcancen las 8 respiraciones por minuto, o menos-, ya que al establecer la respiración en esas frecuencias, el sistema nervioso parasimpático es impactado produciendo profundos estados de relajación y, esa sensación de alivio, incrementando la atención y la claridad mental. ${ }^{17}$ Estas técnicas fueron trabajadas en las aulas de clase, en una frecuencia de dos encuentros semanales y con la intención de indagar, el impacto de dichas técnicas en los estados de ánimo y capacidad de atención/enfoque del alumnado, a nivel individual y a nivel grupal.

\section{Metodología}

Se realizó un estudio mixto, con un diseño cuasiexperimental, de grupo control con medidas pre y posttratamiento, uno grupo de intervención para reducción del estrés con técnicas de pranayama y otro de control. ${ }^{18}$ Además, se realizó un análisis del discurso de los diarios realizados por los estudiantes, proceso escritural que se realizó inmediatamente luego de la práctica de pranayama (estos textos se entregaban en forma anónima, luego de cada sesión).

Para el análisis textual, transcribimos los escritos producidos por los alumnos y los abordamos a través del software NVIVO, con el cual realizamos una búsqueda de la frecuencia de palabras, la cual nos permitió realizar un análisis cualitativo orientado hacia la interpretación de lo manifiesto entre palabras.

En cuanto a las técnicas de respiración, se trabajaron 3 diferentes pranayamas, todos del libro Praana Praanee Praanayam: ${ }^{17}$

1) Sitali praanayam (p.190).

2) Calmándote: incrementando la energía lunar (p.107)

3) El ritmo de la verdad eterna (p.74).

Estas técnicas se ejecutaron, en el contexto del aula aprovechando el espacio y la forma de ser empleadas (sentados, en las sillas del curso). Fueron practicadas entre 5 y 11 minutos, dos días por semana, al inicio, en la mitad o al final de la clase.

\subsection{Selección de los estudiantes criterios de inclusión y exclusión}

La muestra estuvo constituida por 73 estudiantes de primer ciclo de una Universidad Nacional del Ecuador; la distribución de los grupos se conformó de acuerdo a como estaban asignados a cada paralelo: el grupo de intervención con 1 paralelo del primer ciclo $(n=39)$; y el otro formó parte del grupo control $(n=34)$.

Los criterios de inclusión consistían en que sean estudiantes universitarios de primer ciclo de la carrera de Educación General Básica, con deseo de participar que lo expresaron en el consentimiento informado y ser mayores de edad.

\subsection{Instrumento}

El instrumento utilizado fue el Cuestionario de Estrés Académico en la Universidad (CEAU), que evalúa el nivel de estrés que generan las distintas tareas y situaciones académicas. Utiliza una escala licker de 1 a 5 , donde 1 muestra que esa tarea no provoca nada de estrés y 5 que te produce mucho estrés, siendo los números 2, 3 y 4 indican niveles intermedios. ${ }^{19}$

El test se divide en las siguientes escalas:

Obligaciones académicas: evalúa el nivel de estrés relacionado con la realización de tareas, actividades y evaluación en las distintas materias. Comprende elementos como la falta de tiempo para desarrollar las actividades, la sobrecarga académica, la realización de trabajos obligatorios, el exceso de responsabilidades por cumplir con las obligaciones académicas y la realización de exámenes.

Expediente y perspectivas de futuro: mide el nivel de estrés generado por la anticipación de situaciones o potenciales problemas académicos futuros, tales como concluir los estudios en los plazos estipulados, obtener calificaciones elevadas, mantener o conseguir una beca para seguir estudiando, la elección de materias e itinerarios durante la carrera y la presión familiar por obtener buenos resultados.

Dificultades interpersonales: evalúa el nivel de estrés generado por los problemas o conflictos con compañeros y profesores, así como el relacionado con la competitividad académica.

Expresión y comunicación de ideas: mide el nivel de estrés en las situaciones que exigen exponer y expresar ideas propias, ya sea en la presentación de trabajos, la participación e intervención en las actividades de clase o interactuar con los profesores en horario de tutorías.

El instrumento posee una adecuada fiabilidad en cada una de las subescalas, supera el valor de alfa de Cronbach 0.70 , mostrando niveles superiores la dimensión OA $(0.80) .^{19}$

\section{Procedimiento}

Previo al inicio de la investigación se recibió la autorización de los directivos de la Universidad para posteriormente obtener el consentimiento informado de los estudiantes para participar de manera voluntaria, anónima, no remunerada, además se les explico el objetivo del trabajo y que las participaciones no afectaban a sus calificaciones.

Para iniciar, se aplicó el pre-test a todos los estudiantes, lo cual nos permitió evidenciar la presencia del nivel de estrés académico en ambos grupos. Seguido de esto el grupo de intervención inició con las sesiones de prayamana, con la frecuencia de 2 encuentros semanales.

Es necesario puntualizar que se desarrollaron 17 encuentros, en el marco de las clases desde octubre (2018) hasta marzo (2019), de la siguiente manera: 4 en octubre; 4 en noviembre; 2 en diciembre; 4 en enero y 3 en febrero. La cantidad varió por las semanas laborables por mes y, en al- 
gún caso puntual, por interrupción de la jornada por actividades de la Universidad o feriados nacionales/locales.

En la tercera fase, y al finalizar el ciclo académico, se realizó el post test, para posteriormente, realizar los análisis interpretativos del test y de la producción escrita. El grupo de intervención tuvo una pérdida de tres estudiantes porque no completaron la intervención y no cumplimentaron el post-test.

\section{Análisis de resultados}

Se analizaron los datos mediante el programa estadístico SPSS, versión 24. Los datos de medición se expresaron como media \pm desviación estándar, y las comparaciones entre dos grupos se realizaron utilizando la prueba $t$ para muestras independientes, con una significancia estadística de $\mathrm{P}<0.05$.

La producción escrita se analizó a través del programa NVIVO y con la lógica del análisis del discurso.

\section{Resultados}

Los estudiantes presentaron una edad promedio de 19 \pm 2 años; en la tabla 1 se muestran los resultados de las medias y las desviaciones estándar en el pre y post- test de los estudiantes.

Tabla 1: Medias y Desviación estándar en el Pre y Post-Test

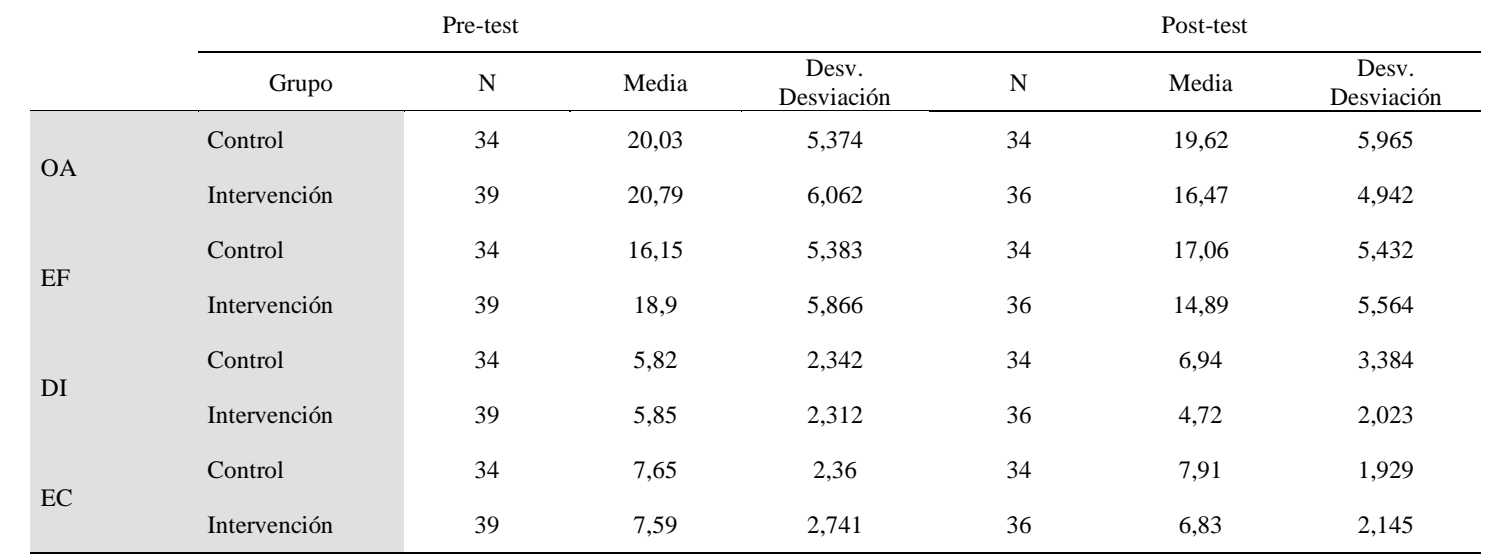

Fuente: Elaboración del autor

Nota: "OA" Obligaciones académicas; "EF" Expediente y expectativas de futuro; "DI"Dificultades interpersonales; "EC" Expresión y comunicación de ideas.

Las medias del grupo de control en la escala de obligaciones académicas, dificultades interpersonales y expresión de las ideas son similares al grupo de intervención en el pre-test.
Además, se pudo observar que las medias de las escalas del test CEAU en el grupo de control pre y post test se mantuvieron o elevaron, a diferencia del grupo de intervención en los que disminuyeron los niveles de estrés (Tabla 1).

Tabla 2: Resultados de la Prueba T de Student en el Pre-Test

\begin{tabular}{|c|c|c|c|c|c|c|c|c|c|}
\hline \multirow[t]{2}{*}{ Escala } & \multirow[t]{2}{*}{$\mathrm{F}$} & \multirow[t]{2}{*}{ Sig. } & \multirow[t]{2}{*}{$\mathrm{t}$} & \multirow[t]{2}{*}{$\mathrm{gl}$} & \multirow[t]{2}{*}{$\mathrm{p}$} & \multirow{2}{*}{$\begin{array}{l}\text { Diferencia de } \\
\text { medias }\end{array}$} & \multirow{2}{*}{$\begin{array}{l}\text { Diferencia de } \\
\text { error estándar }\end{array}$} & \multicolumn{2}{|c|}{$\begin{array}{c}95 \% \text { de intervalo de } \\
\text { confianza de la } \\
\text { diferencia }\end{array}$} \\
\hline & & & & & & & & Inferior & Superior \\
\hline $\mathrm{OA}$ & 0,096 & 0,758 & $-0,567$ & 71 & 0,572 & $-0,765$ & 1,35 & $-3,457$ & 1,926 \\
\hline $\mathrm{EF}$ & 0,002 & 0,964 & $-2,076$ & 71 & 0,042 & $-2,75$ & 1,325 & $-5,392$ & $-0,109$ \\
\hline DI & 0,303 & 0,584 & $-0,041$ & 71 & 0,967 & $-0,023$ & 0,546 & $-1,111$ & 1,065 \\
\hline $\mathrm{EC}$ & 0,804 & 0,373 & 0,095 & 71 & 0,925 & 0,057 & 0,603 & $-1,145$ & 1,26 \\
\hline
\end{tabular}

Fuente: Elaboración del autor

Nota: "OA" Obligaciones académicas; "EF" Expediente y expectativas de futuro; "DI"Dificultades interpersonales; "EC" Expresión y comunicación de ideas. 
Los resultados en el pre-test del grupo de control en las escalas OA $(\mathrm{M}=20,03 \mathrm{SE}=5,374, \mathrm{t}(71)=0,567, \mathrm{p}<.05)$, DI $(\mathrm{M}=5,82 \mathrm{SE}=2,342, \mathrm{t}(71)=0,041 \mathrm{p}<.05), \mathrm{EC}(\mathrm{M}=7,65$ $\mathrm{SE}=2,36, \mathrm{t}(71)=0,095, \mathrm{p}<.05)$, no presentan diferencias significativas iniciales que el grupo de intervención. Mientras que la escala de EF presenta diferencias significativas $(\mathrm{M}=16,15 \mathrm{SE}=5,383, \mathrm{t}(71)=2,076, \mathrm{p}<.05)($ Tabla 2$)$.

Tabla 3: Resultados de la Prueba T de Student en el Post-Test

\begin{tabular}{|c|c|c|c|c|c|c|c|c|c|}
\hline \multirow{2}{*}{ Escala } & \multirow{2}{*}{$\mathrm{F}$} & \multirow{2}{*}{ Sig. } & \multirow{2}{*}{$\mathrm{t}$} & \multirow{2}{*}{$\mathrm{gl}$} & \multirow{2}{*}{$\mathrm{p}$} & \multirow{2}{*}{$\begin{array}{l}\text { Diferencia de } \\
\text { medias }\end{array}$} & \multirow{2}{*}{$\begin{array}{l}\text { Diferencia de } \\
\text { error estándar }\end{array}$} & \multicolumn{2}{|c|}{$\begin{array}{l}95 \% \text { de intervalo de } \\
\text { confianza de la diferencia }\end{array}$} \\
\hline & & & & & & & & Inferior & Superior \\
\hline $\mathrm{EF}$ & 0,376 & 0,542 & 1,65 & 68 & 0,104 & 2,17 & 1,315 & $-0,455$ & 4,795 \\
\hline EC & 0,291 & 0,591 & 2,208 & 68 & 0,031 & 1,078 & 0,488 & 0,104 & 2,053 \\
\hline
\end{tabular}

Fuente: Elaboración del autor

Nota: "OA" Obligaciones académicas; "EF" Expediente y expectativas de futuro; "DI"Dificultades interpersonales; "EC" Expresión y comunicación de ideas.

Los resultados del post-test en los estudiantes, evidencian que el grupo de intervención presenta diferencias significativas en la escala $\mathrm{OA}(\mathrm{M}=16,47 \mathrm{SE}=4,942$ $\mathrm{t}(68)=2,408, \mathrm{p}<, 05)$, DI $(\mathrm{M}=4,72 \mathrm{SE}=2,023 \mathrm{t}(68)=3,352, \mathrm{p}$ $<, 05)$, EC $(\mathrm{M}=6,83 \mathrm{SE}=2,145 \mathrm{t}(68)=2,208, \mathrm{p}<, 05)$, mien- tras que EF no presenta diferencias significativas $(\mathrm{M}=14,89$ $\mathrm{SE}=5,564, \mathrm{t}(68)=1,65, \mathrm{p}<.05)($ Tabla 3$)$.

\subsection{Análisis del discurso}

De la totalidad de palabras transcritas y analizadas, las 9 que mayor frecuencia presentaron, son:

Tabla 4: Palabras con mayor frecuencia

\begin{tabular}{|l|c|c|c|}
\hline \multicolumn{1}{|c|}{ Palabras } & Recuento & Frecuencia & Sinónimos \\
\hline sentí & 77 & $3,26 \%$ & sentir \\
\hline espalda & 35 & $1,48 \%$ & espalda \\
\hline dolor & 33 & $1,40 \%$ & dolor, dolores \\
\hline tranquilidad & 26 & $1,10 \%$ & tranquilidad \\
\hline momento & 23 & $0,97 \%$ & momento, momentos \\
\hline cuerpo & 23 & $0,97 \%$ & cuerpo \\
\hline respiración & 23 & $0,97 \%$ & respiración \\
\hline siente & 21 & $0,89 \%$ & siente, sientes \\
\hline relajación & 18 & $0,76 \%$ & relajación \\
\hline
\end{tabular}

Fuente: Elaboración del autor

A partir de esta frecuencia de repeticiones, abordamos la interpretación de significados rastreando el sentido que los enunciados construyen en el contexto áulico y de la práctica a la que han sido expuestos los alumnos.

Por lo dicho, se observó en primera instancia, que la palabra "sentî" es la que más presencia reporta. Se entiende aquello desde la premisa de escritura en la cual los estudiantes, tras haber experimentado los estadios a los que lleva la meditación profunda, hablan desde la primera persona y vuelcan su búsqueda, consiente o no, hacia los registros que su memoria guarda en relación a procesos emocionales, no racionales.

En cuanto a las relaciones que la palabra "sentí" construye en el discurso de los alumnos, debemos resaltar la presencia, la compañía constante de imágenes que evidencian procesos de relajación, en los vocablos repetitivos con que se completan los enunciados en torno a ese "sentir": "calma"; "alivio"; "tranquilidad"; "paz"; "relajamiento". 
Es muy interesante el reparar en que, las dos palabras que reportan mayor frecuencia luego de la analizada, son "dolor" y "espalda". Se entiende aquello desde la dificultad del alumno por guardar una postura recta, separada del espaldar, por 8 u 11 minutos; el dolor, entonces, fue uno de los principales síntomas, aunque, en casi la totalidad de reportes sobre este malestar, se destaca que, una vez que la respiración se focalizaba, el mismo desaparecía dejando espacio a profundos espacios de confort y relajación.

La siguiente palabra con mayor frecuencia fue "tranquilidad". En los enunciados que con ella se construyen, podemos encontrar vocablos repetitivos como: "paz", "silencio", "interior", "armonía", "concentración", "relajamiento" y "calma".

Las siguientes palabras con mayor presencia son "cuerpo" y "respiración". El vínculo, obvio, lo resaltamos a través de las imágenes y los enunciados que las crean, a partir, nuevamente, de la repetición de vocablos que son conceptos que los alumnos van asumiendo con la práctica. Así, al referirse al cuerpo, encontramos repetitivas imágenes que se relacionan con "experiencia de paz", "tranquilidad", "relajación", "liberación".

Y cuando se refieren a la "respiración", los vocablos/imágenes/conceptos reiterativos hacen relación a, nuevamente, la "relajación", al "momento", la "concentración", al "control" sobre "el dolor y el miedo" y sobre "ruidos".

\section{Discusión}

El estrés es un fenómeno presente en los estudiantes universitarios, ${ }^{8,19}$ como se ha observado en el presente trabajo. Mediante la intervención para reducción del estrés con técnicas de pranayama se pudo evidenciar que los niveles de estrés en el factor de obligaciones académicas, que se relaciona con la falta de tiempo para desarrollar las actividades o la sobrecarga académica, presentó una disminución; al igual que las dificultades interpersonales, que se relacionan con los problemas entre compañeros, profesores; y la comunicación de ideas propias, con la participación de actividades en clase y exposiciones, lo que concuerda con otras investigaciones. ${ }^{6,20-22}$

Mientras que la escala de expediente y perspectivas de futuro, a pesar que se evidencia una disminución del malestar, no es significativamente diferente que el grupo de control; es decir: los estudiantes mantienen una preocupación generada por concluir los estudios en los plazos estipulados, obtener calificaciones elevadas, mantener o conseguir una beca y por la presión familiar.

\section{Conclusiones}

Por otra parte, el análisis sobre la producción textual de los alumnos, nos permite evidenciar una construcción de enunciados que valoran positivamente la experiencia, asociándola con estadios de relajación, paz, liberación, bienestar físico, emocional que les lleva a una conciencia plena del presente.
El interés de este trabajo se centró en comparar estudiantes en los cuales, un grupo realizó una intervención para reducir el estrés con técnicas de pranayama, con una respiración consiente es decir, manejada a voluntad, lo que produce una disminución de los niveles de estrés, lo que concuerda con hallazgos semejantes en otras investigaciones. ${ }^{20,22,23}$

Algunas limitaciones del estudio son las características no probabilísticas de la muestra utilizada, y el tamaño del mismo, ya que una muestra mayor seguramente detectaría diferencias más claras. Sostenemos que una práctica regular de las técnicas de pranayama, en el contexto áulico, son de mucha utili $\neg$ dad en la reducción del estrés académico.

\section{Fuente de Financiamiento}

Este estudio es autofinanciado.

\section{Conflicto de Intereses}

No existen conflictos personales, profesionales, financieroso de otro tipo.

\section{Consentimiento Informado}

Los autores cuentan con el consentimiento informado de los pacientes para la investigación, la publicación del caso y sus imágenes.

\section{Referencias Bibliográficas}

1. Oblitas L. Psicología de la Salud. Cuarta edi ed. México D. F: CENGAGE Lerning; 2017.

2. Peen State University Counseling \& Psychological. Center for Collegiate Mental Health; 2018.

3. American College Health Association. American College Health Association-National College Health Assessment Spring 2007 Reference Group Data Report (Abridged). Journal of American College Health. 2008 mar;56(5):469-480.

4. Parra-Osorio L, Rojas- Castaño L, Patiño- Cordova I, Caicedo-Ausecha G, Valderrama-Aguirre A. Correlación entre el sindrome de Bornout y Estrés Académico en Estudiantes de Medicina de una Facultad de Salud en Cali, Colombia. Rev Colombiana de Salud ocupacional. 2015;3:31-34.

5. Lemos M, Henao-Pérez M, López-Medina D. iMedPub Journals Estrés y Salud Mental en Estudiantes de Medicina : Relación con Afrontamiento y Actividades Extracurriculares Stress and Mental Health in Medical Students : Relation with Coping and Extracurricular Activities Introducción. Archivos de Medicina. 2018;14(2:3):1-8.

6. Castillo C, Chacón T, Diaz-Véliz G. Ansiedad y fuentes de estrés académico en estudiantes de carreras de la salud. investigacion en Educación Mèdica. 2016;5(20):230-237.

7. Dominguez-Lara SA. Academic emotional exhaustion in university students: How much is it influenced by cognitive emotion regulation strategies? Educacion Medica. 2018;19(2):96-103. 
8. Pulido M, Serrano M, Valdés E, Chávez M, Hidalgo P, Vera F. Estrés académico en estudiantes universitarios. 19. Psicología y Salud. 2011;21(1):31-37.

9. Rubin D, C F. A Multicomponet Stress Management Program for College Students. Journal of Counseling and Development. 1986;64:530-531.

10. Álvarez-silva LA, Gallegos-luna RM, Herrera-lópez PS. 20. Estrés académico en estudiantes de Tecnología Superior. Universitas Revista de Ciencias Sociales y Humanas. 2018;(28):193-209.

11. Lilian G. Adaptación escolar y estrés en los estudiantes 21 del primer semestre de la facultad ciencias de la salud, Universidad Nacional de Chimborazo, período 2016; 2016.

12. Quito V, Tamayo M, Buñay P, Neira O. Estrés Academico 22 en Estudiantes de tercero de bachillerato de las unidades educativas particulares del Ecuador. Revista Electronica de Psicologia Iztacala. 2017;20(3):253-276.

13. Cabanach RG, Souto-Gestal A, Franco V. Escala de Estresores Académicos para la evaluación de los estresores académicos en estudiantes universitarios. Revista Iberoamericana de Psicologia y Salud. 2016;7(2):41-50.

14. Becker I. Uses of yoga in psychiatry and medicine. Complementary and alternative medicine and psychiatry. American p ed. Washington; 2000. p. 107-145.

15. Bhajan Y. The Aquarian Teacher KRI: International Teacher Training in Kundalini Yoga as Taught By Yogi Bhajan Level One Instructor Textbook and Instructor Yoga Manual. 4th ed. Training) KKT, editor; 2007.

16. Zaccaro A, Piarulli A, Laurino M, Garbella E, Menicucci D, Neri B, et al. How Breath-Control Can Change Your Life : A Systematic Review on Psycho-Physiological Correlates of Slow Breathing. Frontiers in Human Neuroscience. 2018;12(September):1-16.

17. Bhajan Y. Praana Praanee Praanayam. Kundalini Research Institute; 2006.

18. Hernández Sampieri R, Fernández Collado C, Baptista Lucio MdP. Metodología de la Investigación. Sexta ed.
S A IEdCV, editor. México D. F: McGraw-Hill; 2014.

García-Ros R, Pérez-González F, Pérez-Blasco J, Natividad LA. Evaluación del estrés académico en estudiantes de nueva incorporación a la universidad [Academic Stress Evaluation in New Students Joining University]. Revista Latinoamericana de Psicologia. 2012;44(2):143-154.

Cea Ugarte JI, González-Pinto Arrillaga A, Cabo González $\mathrm{OM}^{\mathrm{a}}$. Efectos de la respiración controlada sobre los síntomas de estrés y ansiedad en una población de 55 a 65 años: estudio piloto. Gerokomos. 2015;26(1):18-22.

Chiesa A, Serretti A. Mindfulness-Based Stress Reduction for Stress Management in Healthy People: A Review and Meta-Analysis. The Journal of Alternative and Complementary Medicine. 2009;15(5):593-600.

. Falsafi N. A Randomized Controlled Trial of Mindfulness Versus Yoga. Journal of the American Psychiatric Nurses Association. 2016;22(6):483-497.

23. Park CL, Riley KE, Braun TD, Jung JY, Suh HG, Pescatello LS, et al. Yoga and Cognitive-behavioral Interventions to Reduce Stress in Incoming College Students: A Pilot Study. Journal of Applied Biobehavioral Research. 2017;22(4):1-26.

Recibido: 13 de enero de 2020

Aceptado: 25 de septiembre de 2020 
